\title{
An Outbreak of Acinetobacter baumannii Septicemia in a Neonatal Intensive Care Unit of a University Hospital in Brazil
}

Denise von Dolinger de Brito ${ }^{1}$, Elias José Oliveira ${ }^{1}$, Vânia O. Steffen Abdallah'², Ana Lúcia da Costa Darini ${ }^{3}$ and Paulo P. Gontijo Filho ${ }^{1}$

\author{
${ }^{1}$ Laboratory of Microbiology, Federal University of \\ Uberlândia; ${ }^{2}$ Neonatal ICU, Clinical Hospital; Uberlândia, \\ $M G$; ${ }^{3}$ Special Laboratory of Bacteriology and Molecular \\ Epidemiology, School of Pharmaceuthical Sciences of \\ Ribeirão Preto, University of São Paulo; Ribeirão Preto, \\ SP, Brazil
}

\begin{abstract}
We studied an outbreak of two multi-drug resistant clones of Acinetobacter baumannii in the Neonatal Intensive Care Unit of the Uberlândia Federal University Hospital in Minas Gerais state, Brazil, and we analyzed the contribution of cross-transmission in the rise in infection rates. Eleven neonates who developed multi-drug resistant $A$. baumannii nosocomial infection were matched to 22 neonates who were admitted to the same unit and did not develop an infection during the outbreak period, in order to identify risk factors for infection. Three out of the 11 neonates died. Epidemiological investigation included molecular typing, using pulsed field gel electrophoresis. Prior to the outbreak, from December 2001 to March 2002, no case of infection by this microorganism was diagnosed. Environmental and healthcare worker hand cultures were negative. Nine isolates had similar pulsed field gel electrophoresis patterns and two had another clone. The first clone was brought into the unit by an infected patient who was transferred from another hospital without a history of antibiotic use. The second clone did have its origin clearly defined. Both infected groups led us to conclude that several factors contributed to infection with A. baumannii. These factors were: exposure to antibiotics and invasive devices, birth weight $\leq$ $1500 \mathrm{~g}$, age $\leq 7$ days and duration of hospitalization $\geq 7$ days. Based on logistic regression, infected neonates were more exposed to carbapenem and mechanical ventilation than the control group. Cross transmission between infants contributed to the rise in the rates of multi-drug resistant $A$. baumannii infection.
\end{abstract}

Key Words: Acinetobacter baumannii, molecular epidemiology, outbreak, neonates.

Acinetobacter baumannii is a ubiquitous microorganism that has become an important nosocomial pathogen, particularly in intensive care units (ICUs) [1,2]. Various types of infections are caused by this microorganism, including pneumonias, bacteremias, meningitis and urinary tract infections [3-

Received on 18 April 2005; revised 27 July 2005.

Address for correspondence: Dr. Denise Von Dolinger de Brito. Avenida Floriano Peixoto, 5322, bloco 09, apt. 302, Residencial Buritis, Umuarama. Zip code: 38405-184, Uberlândia, MG, brazil. Phone (34) 3232-2280. Fax: (34) 3218-2333. E-mail: denisebrito@terra.com.br

The Brazilian Journal of Infectious Diseases 2005;9(3):301-309 (C) 2005 by The Brazilian Journal of Infectious Diseases and Contexto Publishing. All rights reserved.
5]. Although classically described as a nosocomial pathogen in adults, A. baumannii is also an important pathogen in neonates hospitalized in ICUs [3]. Most A. baumannii outbreaks have been traced to environmental sources, such as patient mattresses, air conditioners and mechanical ventilation equipment [6]. The risk factors associated with nosocomial infections due to this microorganism include mechanical ventilation, surgery, and trauma [7]. Increasing rates of $A$. baumannii infections may be due to lapses in infectioncontrol practices. In these situations, "colonization pressure", which is a function of the proportion of patients already colonized or infected with $A$. baumannii, can affect the likelihood of crosstransmission between patients [8]. 
We describe an outbreak of nosocomial infection caused by two multidrug-resistant (MDR) $A$. baumannii clones in a Neonatal Intensive Care Unit (NICU), involving 11 cases. In addition, to analyzing the contribution of cross-transmission, the mean daily point prevalence of patients with MDR A. baumannii infection was quantified, and a case-control study was performed.

\section{Materials and Methods}

\section{Clinical setting}

The NICU of Uberlândia Federal University Hospital has nine beds, reserved for patients requiring intensive care, such as mechanical ventilation and cardiac support.

\section{Design of the study}

We made a case-control study from October 2001 to March 2002; a case was defined as any infant hospitalized in the NICU who developed A. baumannii infection $(n=11)$ confirmed by laboratory exam, and other neonates hospitalized during the same period, with no diagnosis of infectious syndrome $(n=22)$ were the controls. The localization of the cases in the incubators was recorded (Figure 1). An individual record was filled out with the patient's demographic data, along with possible predisposing factors, including gender, birth weight, age, duration of hospitalization, gestational age, apgar score at five minutes, incubator care, use of antibiotics, mechanical ventilation and use of a central or peripheral venous catheter.

\section{$\underline{\text { Microbiological investigation }}$}

All the A. baumannii samples were inoculated in sheep blood agar and incubated at $35^{\circ} \mathrm{C}$. Typical colonies were examined. The genus was identified by Gram staining, cell and colony morphology, oxidation/fermentation test activity, absence of motility and negative oxidase and positive catalase reactions. The species identification of $A$. baumannii was determined by lactose and glucose fermentation, urease activity and hemolysis of sheep blood [9]. Clinical oropharynx and rectal materials were obtained with swabs of all hospitalized neonates on two occasions (December 2001 and January 2002), during the outbreak. Environmental cultures were taken at these times, including samples from incubators, tap water, sink areas, mechanical ventilator, liquid soap, and air; this last through the exposition of petri plates with blood agar and MacConkey agar. Cultures from the hands of most staff working in the NICU were obtained during the same periods.

The antibiotic susceptibilities of the 11 A. baumannii isolates were determined by the disk diffusion method on Mueller-Hinton agar plates, with the following antibiotics: gentamicin, ciprofloxacin, ceftazidime, cefpime, cefpirome, aztreonam, imipenem and ampicillin/sulbactam [10], and the minimum inhibitory concentrations (MICs) of ceftazidime and cefpime were determined by agar dilution technique on MuellerHinton agar plates, with the following ceftazidime and cefepime concentrations: 0.5 to $256 \mu \mathrm{g} / \mathrm{mL}$ [11]. The genotypic analysis of isolated $A$. baumannii was performed by pulsed-field gel electrophoresis (PFGE). The DNA fragments generated by digestion with the restriction enzyme SmaI were analyzed using the Gene Navigator apparatus (Pharmacia Biotech). The criterium for interpreting PFGE patterns was the number of fragment differences compared to the outbreak pattern, as follows: 0 differences was interpreted as part of the outbreak; 2 to 3 differences was probably part of the outbreak; 4 to 6 differences was possibly part of the outbreak; and $\geq 7$ differences was not part of the outbreak [12].

\section{Case-control study}

For each patient, the mean daily point-in-time prevalence of neonates with MDR A. baumannii infection in the unit was calculated. The final point was the date of the discharge from the unit for cases and controls. 


\section{$\underline{\text { Statistical analysis }}$}

Categorical variables were compared by using the likelihood ratio test or, when appropriate, Fisher's exact test. Continuous variables were analyzed by the student's t-test, or the Wilcoxon Rank-Sum Test for non-parametric distributions [13]. To assess the independent effects of significant variables in the univariate analysis, we performed multivariate analysis, using stepwise logistic regression [14].

\section{Results}

Description of the outbreak and case characteristics

The outbreak began in October 2001, and it extended until March 2002 (Figure 2); In the previous year, no case of infection by A. baumannii was recorded in the NICU. In September 2002, a neonate was observed to be infected by $A$. baumannii; however, the microorganism did not have the same characteristics as the epidemic strain. The index case occurred in the first week of October. It involved a $2760 \mathrm{~g}$ neonate, coming from the Maternity Nossa Senhora Aparecida, of Iturama, MG; the baby was transferred at 45 hours after birth, in convulsive crisis, with suspicion of meningitis and without report of antibiotic use. During the outbreak, the epidemic strain of A. baumannii was isolated from 11 neonates. It was identified from blood culture, and the three neonates that died presented the following characteristics (Table 1): case 3 $2610 \mathrm{~g}$, cardiac patient, maintained in an incubator, use of a central venous catheter, cases 10 and 11, infected with the second clone, premature $(1010 \mathrm{~g})$, maintained in incubators, breather use and umbilical venous catheter (case 10) and peripheral catheter (case 11). All the infected neonates were treated with imipenem.

During the period of the outbreak, three beds of the NICU had held more than one infected neonate. The unit was not closed to new admissions.

\section{Epidemiology}

The risk factors for A. baumannii infection are described in the Table 2. On univariate analysis, the following factors were statistically significant: weight $£$ $1500 \mathrm{~g}$, age $\leq 7$ days, duration of hospitalization $\geq 7$ days, carbapenem use, antibiotic use, use of central venous catheter and mechanical ventilation, when compared to the control group. By conditional logistic regression, the only independent risk factors for developing a nosocomial MDR A. baumannii infection were the use of carbapenem (odds ratio, 4.95; 95\% confidence interval, 1.51-18.80; $\mathrm{p}=0.031$ ) and mechanical ventilation (odds ratio, 3.49; $95 \%$ confidence interval, 1.13-15.20; $\mathrm{p}=0.047$ ).

\section{$\underline{\text { Risk factors for A. baumannii infection }}$}

Cases were exposed to a mean of 19.2 different patients (range, 6-41) in the unit, among whom a mean of 3.1 (range, 0-6) neonates had an MDR A. baumannii infection. In contrast, controls were exposed to a mean of 8.8 different patients (range 1-29), of whom an average of 2.9 (range 1-5) neonates had an MDR A. baumannii infection. The mean daily point prevalence of neonates with MDR A. baumannii infections was significantly greater among cases $(28.1 \pm 16.9 \%)$ than among controls $(13.4 \pm 7.4 \% ; \mathrm{p}=0.005)$.

In spite of the outbreak being restricted to six months, there were administrative difficulties in instituting cohort isolation, resulting in the death of three hospitalized neonates, all of them occurring after the detection of the A. baumannii epidemic strain in other neonates of the unit.

Although there was surveillance intestinal and oropharynx colonization, and cultures were made from health care workers' hands and several environmental sources at during two periods (December 2001 and January 2002), both during the epidemic (Figure 2), the results were negative.

Microbiological surveillance

All the A. baumannii isolates presented the same resistance profile to the antibiotics gentamicin, 
Figure 1. Diagram of the neonatal intensive care unit, October 2001, to March 2002.

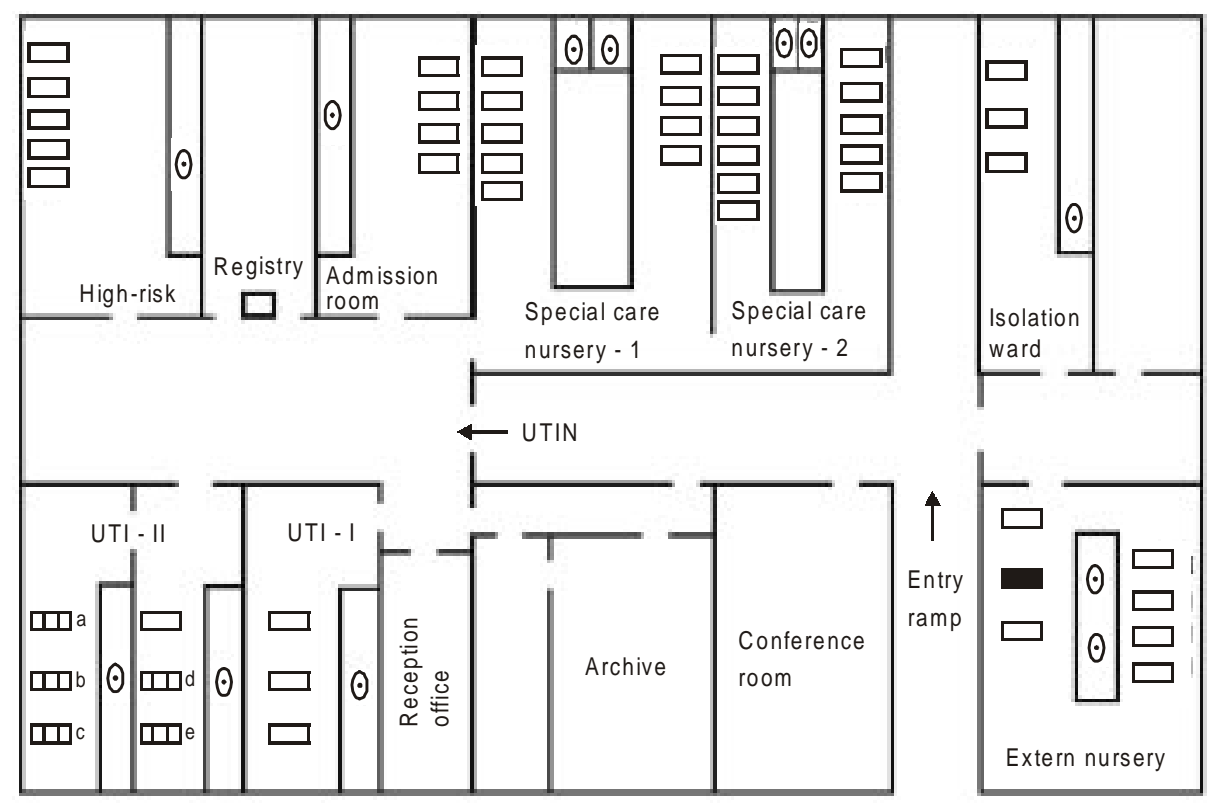

$a=$ case $2 ; b=$ case 1,$3 ; c=$ case $6 ; d=$ case $4,5,7,11 ; e=$ case $8,9,10$

$\odot \operatorname{Sin} k$

पा Incubator with infected neonate

incubator with infected neonate with non-epidemic sample

Figure 2. Distribution of neonatal intensive care unit case patients. Uberlândia Federal University, Uberlândia, Brazil, September, 2001 to September, 2002.

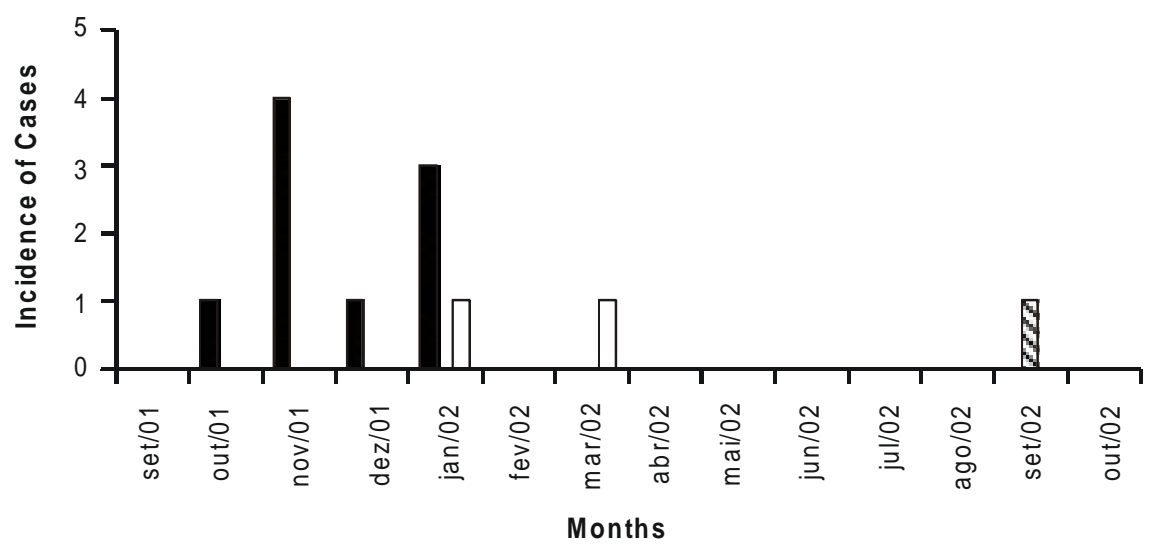

\footnotetext{
Acinetobacter baumannii (epidemic)

$\boldsymbol{\nabla}$ Acinetobacter baumannii (non epidemic)

$\square$ Acinetobacter baumannii (second clone)
} 
Figure 3. Representative pulsed field gel electrophoresis patterns from 11 strains of the Acinetobacter baumannii.

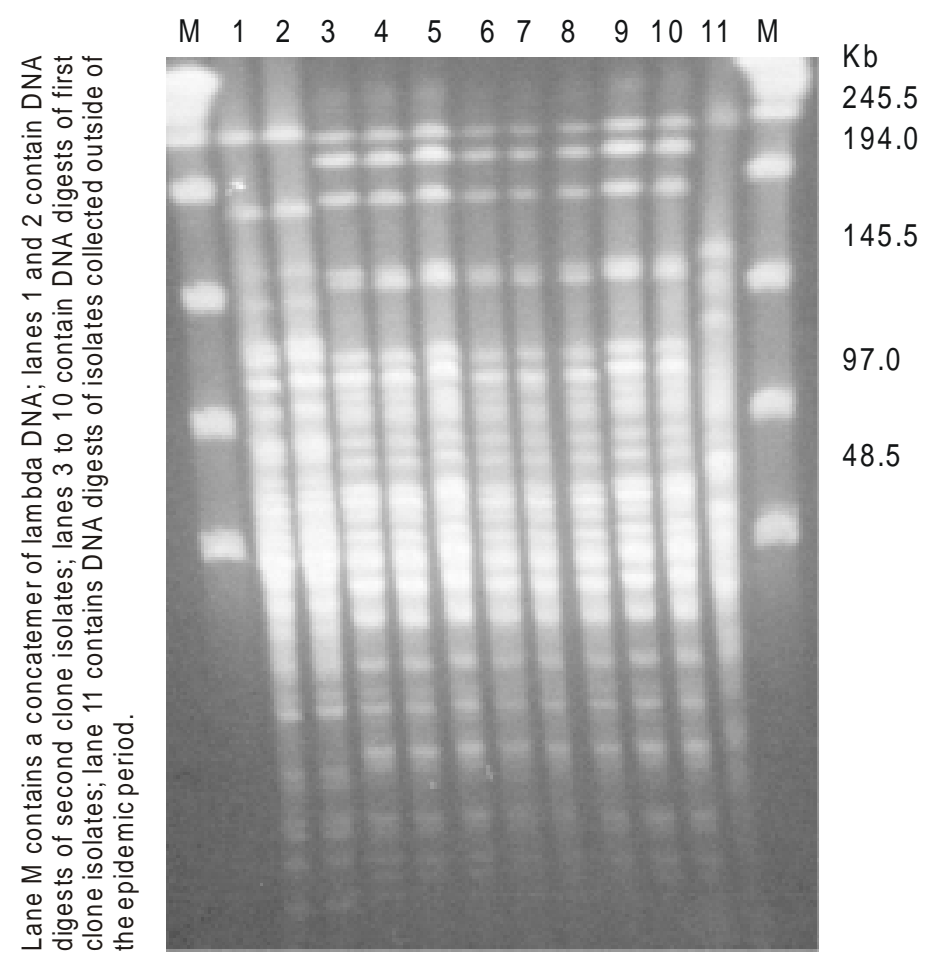

ciprofloxacin, ceftazidime, cefpime and aztreonam, with susceptibility only to ampicillin/sulbactam and imipenem. The epidemic strain was resistant to ceftazidime and cefpime by the agar dilution test. Production of ESBL was detected in six of the 11 samples (data not shown).

Analysis of the A. baumannii strains with PFGE showed two clones, both with band profiles different from the strain of this microorganism that was isolated in the unit after the outbreak (September 2002). The PFGE patterns of the 11 strains of the A baumannii isolates are shown in Figure 3; lane M contains concatemers of lambda DNA; lanes 1 and 2 contain DNA digests of second clone isolates; lanes 3 to 10 contain DNA digests of first clone isolates; lane 11 contains DNA digests of isolates from outside of the epidemic period.

\section{Discussion}

The bacteria A. baumannii has emerged as an important nosocomial pathogen, and outbreaks due to multiply-resistant strains have been difficult to control, especially in ICUs [15]. We have documented an outbreak of MDR A. baumannii in an NICU during a six-month period, affecting 11 neonates, with three deaths. To define the risk factors for the acquisition of infection, we compared the case-infant group with a randomly-selected group of control infants; the following factors were significant: weight $\leq 1500 \mathrm{~g}$, age $\leq 7$ days, duration of hospitalization $\geq 7$ days, use of imipenem, use of a central venous catheter and mechanical ventilation. These same risk factors were described by McDonald [3] and Melamed [5].

Although the outbreak caused by $A$. baumannii was traced to a common source, in agreement with prior studies, the environmental reservoirs indicated in those studies (respiratory equipment [2], mattresses [3], suction catheters [2], laryngoscopes [2], gloves [6] and air conditioner [3] were not identified. Though the dissemination of this pathogen is facilitated by its prolonged survival on inanimate surfaces, high colonization rates among hospitalized patients, and frequent contamination of healthcare workers' hands 


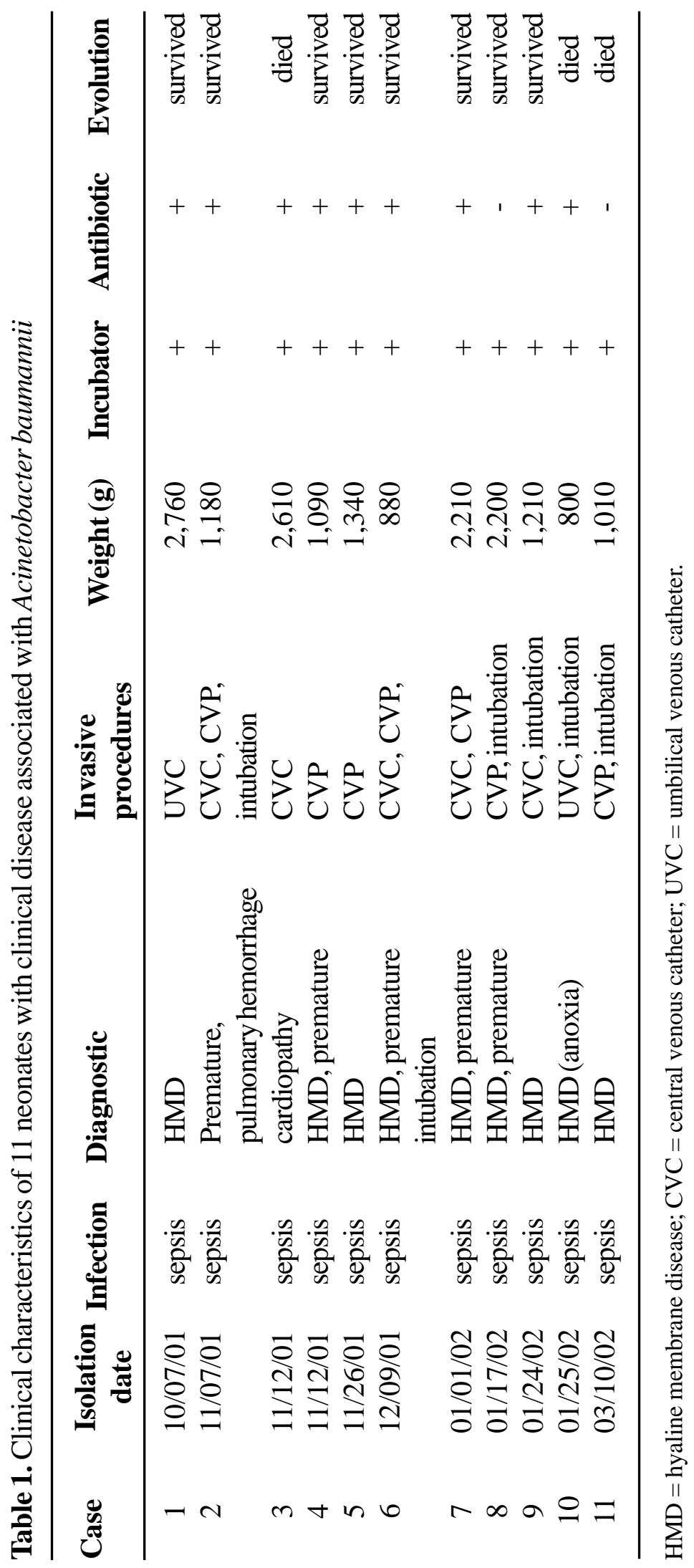


Table 2. Risk factors for Acinetobacter baumannii infection in neonates

\begin{tabular}{|c|c|c|c|c|c|c|}
\hline \multirow[t]{2}{*}{ Risk factor } & \multicolumn{2}{|c|}{$\begin{array}{l}\text { Cases } \\
\mathrm{N}=11 \\
\end{array}$} & \multicolumn{2}{|c|}{$\begin{array}{c}\text { Controls } \\
\mathrm{N}=22 \\
\end{array}$} & \multirow[t]{2}{*}{ Unadjusted OR } & \multirow[t]{2}{*}{ p Value } \\
\hline & $\mathbf{N}$ & $\%$ & $\mathbf{N}$ & $\%$ & & \\
\hline \multicolumn{7}{|l|}{ Weight at birth } \\
\hline$>1500 \mathrm{~g}$ & 4 & 3.6 & 17 & 77.3 & $0.17(0.02-1.03)$ & $0.05^{*}$ \\
\hline$\leq 1500 \mathrm{~g}$ & 7 & 69.6 & 5 & 22.7 & & \\
\hline \multicolumn{7}{|l|}{ Sex } \\
\hline Female & 8 & 72.3 & 7 & 31.8 & $5.71(0.93-39.7)$ & 0.06 \\
\hline Male & 3 & 27.3 & 15 & 68.2 & & \\
\hline \multicolumn{7}{|l|}{ Age } \\
\hline$>7$ days & 7 & 63.6 & 21 & 95.4 & $0.08(0.00-1.06)$ & $0.03 *$ \\
\hline$\leq 7$ days & 4 & 36.4 & 1 & 4.6 & & \\
\hline \multicolumn{7}{|l|}{ Weeks of gestation } \\
\hline $26-39$ & 5 & 45.4 & 4 & 18.2 & $3.75(0.59-25.79)$ & 0.12 \\
\hline $30-33$ & 4 & 36.4 & 6 & 27.3 & $1.52(0.25-9.29)$ & 0.69 \\
\hline $34-36$ & 2 & 18.2 & 12 & 54.5 & $0.19(0.02-1.30)$ & 0.07 \\
\hline $\begin{array}{l}\text { Hospitalization duration } \\
\text { ( } \geq 7 \text { days) }\end{array}$ & 10 & 90.9 & 6 & 27.3 & $26.67(2.41-692.79)$ & $<0.001 *$ \\
\hline \multicolumn{7}{|l|}{ Apgar at 5 minutes } \\
\hline $0-4$ & 4 & 36.4 & 5 & 22.7 & $1.94(0.31-12.50)$ & 0.43 \\
\hline $5-7$ & 1 & 9.1 & 7 & 31.8 & $0.21(0.01-2.31)$ & 0.21 \\
\hline $8-10$ & 6 & 54.5 & 10 & 45.5 & $1.44(0.27-7.87)$ & 0.90 \\
\hline Incubator & 11 & 100.0 & 20 & 20.9 & indefinite & 0.54 \\
\hline Antibiotic use & 11 & 100.0 & 12 & 54.5 & indefinite & $0.01 *$ \\
\hline Carbapenem use & 11 & 100.0 & 0 & 0 & indefinite & $<0.001 *$ \\
\hline Utilization of CVC & 5 & 45.4 & 1 & 4.5 & $17.50(1.42-486.05)$ & $<0.001 *$ \\
\hline Mechanical ventilation & 8 & 72.7 & 1 & 4.5 & $56.00(4.07-1781.29)$ & $<0.001 *$ \\
\hline Daily point prevalence of & $0-20$ & 4 & 15 & & $4.31(1.46-13.00)$ & $0.002 *$ \\
\hline patients with MDR & $21-40$ & 5 & 7 & & & \\
\hline \multirow[t]{3}{*}{ A. baumannii infections (\%) } & $41-60$ & 2 & 0 & & & \\
\hline & $61-80$ & 0 & 0 & & & \\
\hline & $81-100$ & 0 & 0 & & & \\
\hline Exposure to patients with an & 0 & 1 & 0 & & $2.00(0.26-15.60)$ & 0.43 \\
\hline MDR A. baumannii infection & $1-2$ & 3 & 11 & & & \\
\hline during unit admission & $3-4$ & 5 & 4 & & & \\
\hline \multirow[t]{2}{*}{ (number patients) } & $5-6$ & 2 & 7 & & & \\
\hline & $7-8$ & 0 & 0 & & & \\
\hline
\end{tabular}

* $\mathrm{P}=0.05$ (statistically significant); $\mathrm{OR}=$ odds ratio; $\mathrm{MDR}=$ multidrug resistant; $\mathrm{CVC}=$ central venous catheter. 
have been observed. In our study, all environmental and hand cultures were negative for $\operatorname{MDR} A$. baumannii. Another unusual aspect was that the index case was a neonate with suspicion of meningitis, transferred from a small nearby city, without indication of the use of any antibiotics, with a blood culture made before instituting antibiotic therapy. All documented infections in this outbreak were septicemias in neonates using central or peripheral venous catheters. The mortality associated with this infectious syndrome in neonates ranges from 13.9 to $83 \%$ [2,3], while we found $27 \%$ (3 of 11).

The potential for acquisition of MDR A. baumannii from another neonate was considered and quantified by calculating the mean daily point prevalence of neonates with MDR A. baumannii infections to which each neonate was exposed to. Several studies have found that the prior use of third-generation cephalosporins (especially ceftazidime), fluorquinolones, and carbapenems is associated with the subsequent development of MDR A. baumannii $[1,8,12,16,17]$. During the period of March to September 2001, preceding the outbreak that we monitored, an increase in the consumption of this cephalosporin was observed due to an outbreak of Pseudomonas aeruginosa (data not shown).

The A. baumannii isolates were resistant to third and fourth generation cephalosporins, and six out 11 were ESBL producers. Other mechanisms of acquiring resistance have been described for A. baumannii resistant to cephalosporins and carbapenems, including altered penicillin-binding proteins, the presence of metallo beta lactamases, and the loss of porins [17].

Genotyping by PFGE has been shown to be a powerful tool for a better understanding of the epidemiology of nosocomial infection [1,5]. We found two clones of A. baumannii isolated from the blood of neonates hospitalized in our NICU during the study period. Surveillance made during nine-month periods prior and posterior to the outbreak, demonstrated that this microorganism was not present in the unit.

In conclusion, based on a thorough clinical epidemiological investigation, with the use of molecular epidemiology technology, we can conclude that, in many cases, when a contaminated environmental source cannot be identified, the increase in the infection rate due to a particular pathogen may be due to lapses in infection-control measures, resulting in an increase in cross-transmission between patients. Containment of the outbreak was achieved by strict hygienic measures and cohort nursing of the infected infants.

\section{Acknowledgements}

We thank Josiane Ferreira for technical assistance during molecular assays.

\section{References}

1. Wang S.H., Sheng W.H., Chang Y.Y., et al. Healthcare associated outbreak due to pan-drug resistant Acinetobacter baumannii in a surgical intensive care unit. J Hosp Infect 2003;53:97-102.

2. Pillay T., Pillay D.G., Adhikari M., et al. An outbreak of neonatal infection with Acinetobacter linked to contaminated suction catheters. J Hosp Infect 1999;43:299-304.

3. McDonald L.C., Walker M., Loretta C., et al. Outbreak of Acinetobacter spp. bloodstream infections in a nursery associated with contaminated aerosols and air conditioners. Pediatr Infect Dis J 1998; 17:716-22.

4. Bergogne-Berezein E., Towner K.J. Acinetobacter spp. as nosocomial pathogens: microbiological, clinical, and epidemiological features. Clin Microbiol Rev 1996;9:148-65.

5. Melamed R., Greenberg D., Porat N., et al. Successful control of an Acinetobacter baumannii outbreak in a neonatal intensive care unit. J Hosp Infect 2003;53:31-8.

6. Ayan M., Durmaz R., Aktas E. Bacteriological, clinical and epidemiological characteristics of hospital-acquired Acinetobacter baumannii infection in a teaching hospital. J Hosp Infect 2003;54,39-45.

7. Parvez F.M., Jarvis W.R. Nosocomial infections in the nursery. Semin Pediatr Infect Dis 1999;10:119-29.

8. D'Agata E.M.C., Trayer V., Schaffner W. An outbreak of Acinetobacter baumannii: the importance of crosstransmission. Infect Control Hosp Epidemiol 2000;21:588-91.

9. Koneman E.W., Allen S.D., Janda W.M., et al. Bacilos Gram-negativos não-fermentadores. Diagnóstico Microbiológico. 5th edn. Medsi, Rio de Janeiro, 263279, 2001. 
10. National Committee for Clinical Laboratory Standards. Performance standards for antimicrobial disk susceptibility. Approved standard M2-A5, Villanova, PA, USA. NCCLS 2000a.

11. National Committee for Clinical Laboratory Standards. Methods for dilution antimicrobial susceptibility tests for bacteria that grow aerobically. Approved standard M7-A5, Villanova, PA, USA. NCCLS 2000b.

12. Tenover F.C., Arbeit R.D., Goering R.V., et al. Interpreting chromosomal DNA restriction patterns produced by pulsed-field gel electrophoresis criteria for bacterial strain typing. J Clin Microbiol 1995;33:2233-9.

13. Dean A.G. Epi Info, Version 5.0: A word processing, database, and statistics program for epidemiology on microcomputers. Stone Mountain, GA: USD, Ins., 1995.

14. SPSS software PC version 11.0 (SPSS, Chicago).

15. Levin A.S., Levy C.E., Manrique A.E.I., et al. Severe infections with imipenem-resistant Acinetobacter baumannii treated with ampicillin/sulbactam. Intern J Antim Agents 2003;21:58-62.

16. Pillay T., Pillay D.G., Adhikari M., Sturm A.W. Piperacillin Tazobactam in the treatment of Klebsiella pneumoniae infections in neonates. Amer J Perinatol 1998; 15:47-51.

17. Hsueh P.R., Teng L.J., Chen C.Y., et al. Pandrug resistant Acinetobacter baumannii causing nosocomial infections in a university hospital, Taiwan. Emerg Infect Dis 2002;8:827-32. 